\title{
3D deconvolution in Fourier integral microscopy
}

Stefanoiu, Anca, Scrofani, Gabriele, Saavedra, Genaro, Martínez-Corral, Manuel, Lasser, Tobias

Anca Stefanoiu, Gabriele Scrofani, Genaro Saavedra, Manuel MartínezCorral, Tobias Lasser, "3D deconvolution in Fourier integral microscopy," Proc. SPIE 11396, Computational Imaging V, 113960I (18 May 2020); doi: $10.1117 / 12.2558516$

Event: SPIE Defense + Commercial Sensing, 2020, Online Only, California, United States 


\title{
3D deconvolution in Fourier integral microscopy
}

\author{
Anca Stefanoiu ${ }^{\mathrm{a}}$, Gabriele Scrofani ${ }^{\mathrm{b}}$, Genaro Saavedra ${ }^{\mathrm{b}}$, Manuel Martínez-Corral ${ }^{\mathrm{b}}$, and Tobias \\ Lasser $^{\mathrm{a}}$ \\ aDepartment of Informatics, Technical University of Munich, Germany \\ ${ }^{\mathrm{b}}$ Optics Department, University of Valencia, Spain
}

\begin{abstract}
Fourier integral microscopy (FiMic), also referred to as Fourier light field microscopy (FLFM) in the literature, was recently proposed as an alternative to conventional light field microscopy (LFM). FiMic is designed to overcome the non-uniform lateral resolution limitation specific to LFM. By inserting a micro-lens array at the aperture stop of the microscope objective, the Fourier integral microscope directly captures in a single-shot a series of orthographic views of the scene from different viewpoints. We propose an algorithm for the deconvolution of FiMic data by combining the well known Maximum Likelihood Expectation (MLEM) method with total variation (TV) regularization to cope with noise amplification in conventional Richardson-Lucy deconvolution.
\end{abstract}

Keywords: light field microscopy, Fourier integral imaging, deconvolution, 3D reconstruction, expectation maximization, penalized likelihood, regularization, total variation

\section{INTRODUCTION}

Plenoptic (microlens-based) imaging ${ }^{1}$ has been developing based on the principles of integral photography proposed by G. Lippmann in $1908,{ }^{2}$ in both photography ${ }^{3-7}$ and microscopy, ${ }^{8-11}$ and it now represents a hot topic in computational imaging. A conventional microscope can be turned into a light field microscope by strategically inserting a micro-lens array (MLA) into the optical path, in front of the camera sensor, such that both the spatial and angular light field information is captured in a single shot. ${ }^{8}$ Similar to light field photography, such an arrangement allows for various post-acquisition processing possibilities like image refocusing, ${ }^{3,12,13}$ depth estimation ${ }^{14-16}$ or 3D object information retrieval. ${ }^{17-19}$ Light field microscopy (LFM) has proven very attractive around applications involving recording highly dynamic biological processes due to its ability to capture the 3D scene at camera frame rate, without scanning. Demonstrated biological applications include neuro-imaging of fluorescent vertebrate organisms ${ }^{20-22}$ and live cell imaging. ${ }^{23}$

Although LFM has gained great popularity for its scan-less 3D feature, the microscope captures axial information at the expense of lateral resolution. This drawback represents the well-known resolution trade-off in light field imaging. ${ }^{4-8,8,12,17,24}$ The initial accompanying methods for retrieving the $3 \mathrm{D}$ scene from a captured light field image were able to render volumes with lateral resolution limited by the count of micro-lenses in the MLA and axial resolution given by the number of camera pixels behind a micro-lens. More recently, a considerable research effort exploits the depth-dependent light field sampling rates of the plenoptic imaging devices in order to improve the recoverable resolution of the 3D imaged scene, via means of computational super-resolution. ${ }^{7,17,18,25}$ However, due to the depth-varying nature of the sampling patterns of the LFM, the recoverable lateral resolution is non-uniform across depth and it remains low for some axial ranges, especially around the native object plane of the original LFM configuration. ${ }^{17,18}$ On the other hand, hardware variations and extensions were exploited to address these challenges in LFM. Recent works ${ }^{18,23}$ discuss the relative placement of the MLA in the optical path of the LFM, with respect to the camera sensor in order to manipulate the sampling patterns. Similar approaches were employed in light field photography as well. ${ }^{5,6}$ Other proposals include wave-front coding techniques for extended depth of field in LFM, ${ }^{26}$ simultaneous light field and wide field acquisition systems ${ }^{27}$ or dual-LFM devices which combine complementary acquisition parameters. ${ }^{21}$ While all these proposals target the limitations

Further author information: (Send correspondence to A. Stefanoiu)

E-mail: anca.stefanoiu@tum.de

Computational Imaging V, edited by Lei Tian, Jonathan C. Petruccelli,

Chrysanthe Preza, Proc. of SPIE Vol. 11396, 113960I · (C) 2020 SPIE

CCC code: $0277-786 \mathrm{X} / 20 / \$ 21 \cdot$ doi: $10.1117 / 12.2558516$

Proc. of SPIE Vol. 11396 113960I-1 
of the LFM to some extent, they usually mitigate the problems and come at further costs like computationally expensive complementary algorithms or less flexible hardware.

Fourier integral microscopy (FiMic) was recently introduced ${ }^{9}$ to address the current challenges in conventional LFM (or integral microscopy). A conventional wide field microscope can be turned into a Fourier integral microscope (FiMic) by inserting a MLA at the aperture stop of the microscope objective of the host wide field microscope. This arrangement allows for the camera sensor to directly record orthographic views of the imaged sample behind each micro-lens in the array and provides extended depth of field and improved uniform lateral resolution compared to LFM. ${ }^{28}$ In Guo et al. ${ }^{29}$ the authors proposed deconvolution of FiMic images by a wave-based light field point spread function for fluorescent samples, and Cong et al. ${ }^{30}$ demonstrate impressive resolution in 3D imaging of neuro-activity in fish larvae using a customized setup based on the Fourier integral imaging principles.

In this work, we propose a modified deconvolution method to address the noise amplification in the state of the art Richardson-Lucy ${ }^{31,32}$ deconvolution in FLFM. ${ }^{29}$ The iterative update of the optical Richardson-Lucy deconvolution is based on the well-known Maximum Likelihood Expectation Maximization (MLEM) algorithm. ${ }^{33,34}$ The modification we introduce here is based on the penalized likelihood method from. ${ }^{35}$ We employ a total variation $^{36}$ minimization penalty on the solution, to preserve the sharp details, while removing noise. ${ }^{37} \mathrm{We}$ evaluate the proposed method on experimental FiMic images of the USAF-1951 resolution target.

\section{THE FOURIER INTEGRAL MICROSCOPE}

A FiMic is built by inserting a micro-lens array (MLA) at the back aperture stop (AS) of a conventional microscope objective (MO). The camera sensor then captures, behind each of the micro-lenses, a collection of views (elemental images) of the imaged object sample.

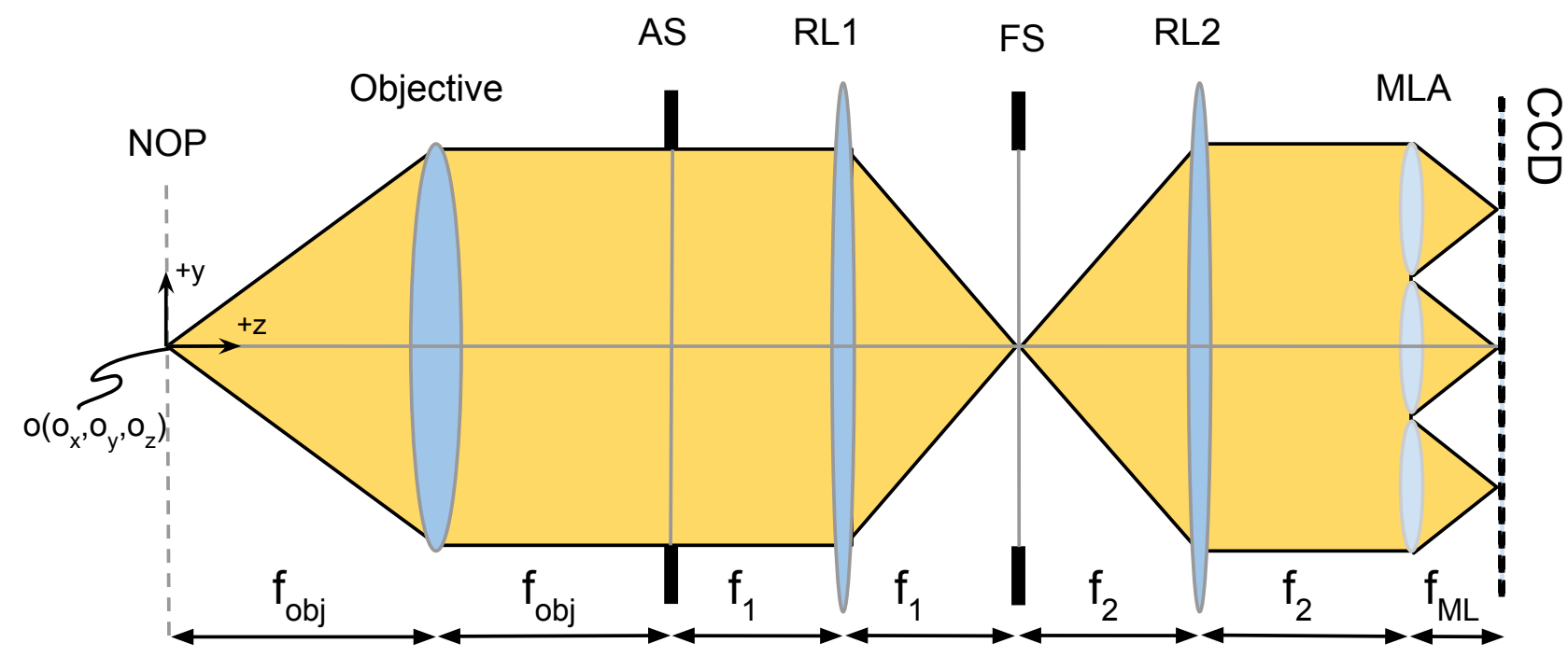

Figure 1. Image formation in FiMic: The microscope illustrated here matches our experimental setup and makes use of an optical relay system (RL1 and RL2 with focal lengths $f_{1}$ and $f_{2}$ ) to conjugate the back aperture (AS) of the microscope objective (MO) with the MLA. While in theory the MLA is inserted at the AS of the MO, in practice the back aperture is usually not accessible in commercial objectives, so we use a relay system to circumvent this limitation. A source point $\mathbf{o}\left(o_{x}, o_{y}, o_{z}\right)$ in front of the MO has a conjugate image by the RL1 lens. Then the RL2 lens picks up this image and elemental images (EIs) are formed behind each micro-lens as the light propagates to the camera sensor plane (CCD). When $f_{1} \neq f_{2}$, the relay system introduces extra magnification to the system. $f_{o b j}$ represents the MO focal length. The field stop (FS) determines both the size of the EIs and of the microscope's field of view. The FS ensures optimal sensor coverage with overlapping of the EIs. 
Figure 1 shows a ray diagram to describe the forward light propagation from a point source in front of the MO to the camera sensor plane and introduces the key components of the FiMic. For practical reasons we use an optical relay system (formed by the two lenses RL1 and RL2) to conjugate the AS plane with the MLA plane, as the AS is usually not mechanically accessible in commercially available microscope objectives. The relay may also contribute to the total system magnification when the focal lengths do not match, $f_{1} \neq f_{2}$. Moving further on the diagram, the size of the field stop (FS) controls the size of the elemental images (EIs) and a properly sized FS ensures the EIs optimally cover the sensor plane, without overlapping. Implicitly, the FS controls the field of view (FOV) of the FiMic as well.

A source point, $\mathbf{o}\left(o_{x}, o_{y}, z\right)$ at the native object plane (NOP) in front of the microscope, has a conjugate image by the first relay lens (RL1), at the field stop plane (FS). This intermediate image is then picked up by the second relay lens, RL2, and magnified images of the FS, formed behind each micro-lens are recorded by the camera as the light propagates to the sensor plane.

\section{DECONVOLUTION}

In this section we describe the reconstruction process of computing a $3 \mathrm{D}$ volume from a raw light field image. Starting from the raw noisy light field sensor measurements $\mathbf{m}=\left(m_{j}\right)_{j \in J}$ acquired by pixels $j \in J$ (where $|J|=m$ ) we aim at retrieving the fluorescence intensity at each discrete point in the imaged 3D object that produced the measurements. We represent the discretized object volume $\mathbf{u}$ by a vector of coefficients $\left(u_{i}\right)_{i \in I}$ with $|I|=n$.

\subsection{Expectation maximization algorithm}

Due to the low photon counts in fluorescence microscopy, we define the number of photons emitted at voxel $i$ and detected by sensor element $j$ as random variables $z_{j i}$ with $z_{j i} \sim \operatorname{Poisson}\left(u_{i} a_{j i}\right)$, which we combine into the the iid random vector $\mathbf{z}=\left(z_{j i}\right)_{j \in J, i \in I}$, with $a_{j i}$ denoting the detection probabilities:

$$
a_{j i}=P(\text { photon detected at sensor element } j \mid \text { photon emitted from voxel } i) .
$$

Then our measurements $\mathbf{m}=\left(m_{j}\right)_{j \in J}$ arise from $z_{j i}$ as $m_{j}=\sum_{i \in I} u_{i} a_{j i}$ yielding the stochastic imaging model:

$$
\mathbf{m} \sim \operatorname{Poisson}(A \mathbf{u})
$$

where the operator $A=\left(a_{j i}\right)_{j \in J, i \in I}$ describes the light field forward propagation model. In this paper, we use a diffraction-aware light propagation model, similar to the one introduced in Guo et al. ${ }^{29}$ Conversely, a previously measured light field point spread function, as in Cong et al. ${ }^{30}$ could be employed. A detailed description of such forward models is out of the scope of this work and we refer the interested reader to the various literature on light field forward models. ${ }^{17,18,26,29,38}$

If we regard $\mathbf{z}$ as the complete version of the incomplete data $\mathbf{m}$, the Expectation Maximization (EM) approach $^{31,33,34}$ aims at finding the maximum likelihood estimate $\mathbf{u}_{M L}$ for the unknown $\mathbf{u}$, that maximizes the joint probability density (likelihood):

$$
l(\mathbf{z} \mid \mathbf{u})=\prod_{j \in J} \prod_{i \in I} e^{-u_{i} a_{j i}} \frac{\left(u_{i} a_{j i}\right)^{z_{j i}}}{z_{j i} !} .
$$

For convenience, we consider the estimation of $\mathbf{u}$ by maximizing the alternative Poisson log-likelihood:

$$
L(\mathbf{u})=\ln l(\mathbf{z} \mid \mathbf{u})=\sum_{j \in J} \sum_{i \in I} u_{i} a_{j i}+z_{j i} \ln x_{i} a_{j i}-\ln z_{j i} !
$$

The EM algorithm provides an iterative two-step scheme for increasing the likelihood of the current estimate, $\mathbf{u}$. In the first step, $\mathbf{z}$ is estimated by computing the conditional expectation $E\left(z_{i j} \mid \mathbf{m}, \mathbf{u}\right)$, and in the second step, the maximum likelihood estimate of $\mathbf{u}$ is found, starting from an initial guess $\mathbf{u}^{0}$ : 


$$
\begin{gathered}
\hat{z_{j i}}=m_{j} \frac{u_{i}^{k} a_{j i}}{\sum_{l \in I} u_{l}^{k} a_{j l}} \\
u_{i}^{k+1}=\frac{u_{i}^{k}}{\sum_{j \in J} a_{j l}} \sum_{j \in J} \frac{m_{j} a_{j i}}{\sum_{l \in I} u_{l}^{k} a_{j l}}
\end{gathered}
$$

In matrix-vector notation, the well known MLEM (Richardson-Lucy ${ }^{31}$ ) iterative update scheme reads:

$$
\mathbf{u}^{k+1}=\frac{\mathbf{u}^{k}}{A^{T} \mathbf{1}}\left[A^{T} \frac{\mathbf{m}}{A \mathbf{u}^{k}}\right]
$$

\subsection{Penalized likelihood}

If we further assume that we have prior information about our solution, it is appropriate to estimate $\mathbf{u}$ by maximizing the penalized log-likelihood, ${ }^{35} L(\mathbf{u})-\lambda J(\mathbf{u})$ instead:

$$
\underset{\mathbf{u}}{\operatorname{argmax}} \sum_{j \in J} \sum_{i \in I} u_{i} a_{j i}+z_{j i} \ln x_{i} a_{j i}-J(\mathbf{u}),
$$

where the prior $J(\mathbf{u})$ could, for example, represent a roughness functional and $\lambda$ a smoothing parameter, as we will see in the next section.

To find a maximizer of the penalized likelihood, we adopt the explicit one step late (OSL) algorithm, ${ }^{35}$ and the iterative update scheme in eq. 7 now becomes:

$$
\mathbf{u}^{k+1}=\frac{\mathbf{u}^{k}}{A^{T} \mathbf{1}+\lambda *\left(\mathbf{D} J(\mathbf{u})_{k}\right)}\left[A^{T} \frac{\mathbf{m}}{A \mathbf{u}^{k}}\right]
$$

where $\mathbf{D}$ denotes the derivative operator.

\subsection{Total variation regularization}

In this section we assume a smoothness requirement on the discrete solution $\mathbf{u}$. Rudin et al. ${ }^{36}$ introduced the total variation (TV) regularization for imaging problems to preserve sharp structures, while removing noise in the images. The roughness penalty functional, defined by the TV of the solution $\mathbf{u}$ is:

$$
J(\mathbf{u})=|\mathbf{u}|_{\mathrm{TV}} .
$$

Here we employ the anisotropic discretization of the TV norm: ${ }^{39}$

$$
|\mathbf{u}|_{\mathrm{TV}}=\sum_{i}\left(\left|\left(\nabla_{x} \mathbf{u}\right)_{i}\right|+\left|\left(\nabla_{y} \mathbf{u}\right)_{i}\right|\right)
$$

where $\nabla_{x} \mathbf{u}$ and $\nabla_{y} \mathbf{u}$ are the horizontal and vertical derivatives, which are discretized here as one-sided forward differences. ${ }^{40}$

When minimizing the negative penalized $\log$-likelihood, $-L(\mathbf{u})+\lambda J(\mathbf{u}))$, the minimizer of the $J(\mathbf{u})$ functional satisfies the corresponding Euler-Lagrange equation, $\operatorname{div} \frac{\nabla \mathbf{u}}{|\nabla \mathbf{u}|}=0$ and the update scheme of the OSL algorithm with TV regularization finally reads:

$$
\mathbf{u}^{k+1}=\frac{\mathbf{u}^{k}}{A^{T} \mathbf{1}+\lambda * \operatorname{div}\left(\frac{\nabla \mathbf{u}}{|\nabla \mathbf{u}|}\right)_{k}}\left[A^{T} \frac{\mathbf{m}}{A \mathbf{u}^{k}}\right]
$$




\section{RESULTS}

We evaluate the proposed deconvolution method using experimentally acquired images of the USAF-1951 resolution target with our custom-built FiMic containing a MLA with $f_{m l}=6,5 \mathrm{~mm}, \mu_{\text {lens }}=1.0 \mathrm{~mm}$ (AMUS APH-Q-P1000-R2.95) and an infinity corrected MO $\left(f_{o b j}=9.0 \mathrm{~mm}\right.$ and $\left.N A_{o b j}=0.4\right)$. The optical relay system consisted of the RL1 and RL2 lenses with focal lengths $f_{1}=125 \mathrm{~mm}$ and $f_{2}=200 \mathrm{~mm}$, respectively. The images were recorded with a CMOS camera (EO-5012c 1/2") with pixel pitch $\rho_{p x}=2,2 \mu m$.

Figure 2 shows a raw light field image, the zoomed-in central EI and a close-up on groups 6 and 7 of the target. The raw image resolves up to element 6.4, when we check for the existence of an intensity dip of about $25 \%$.
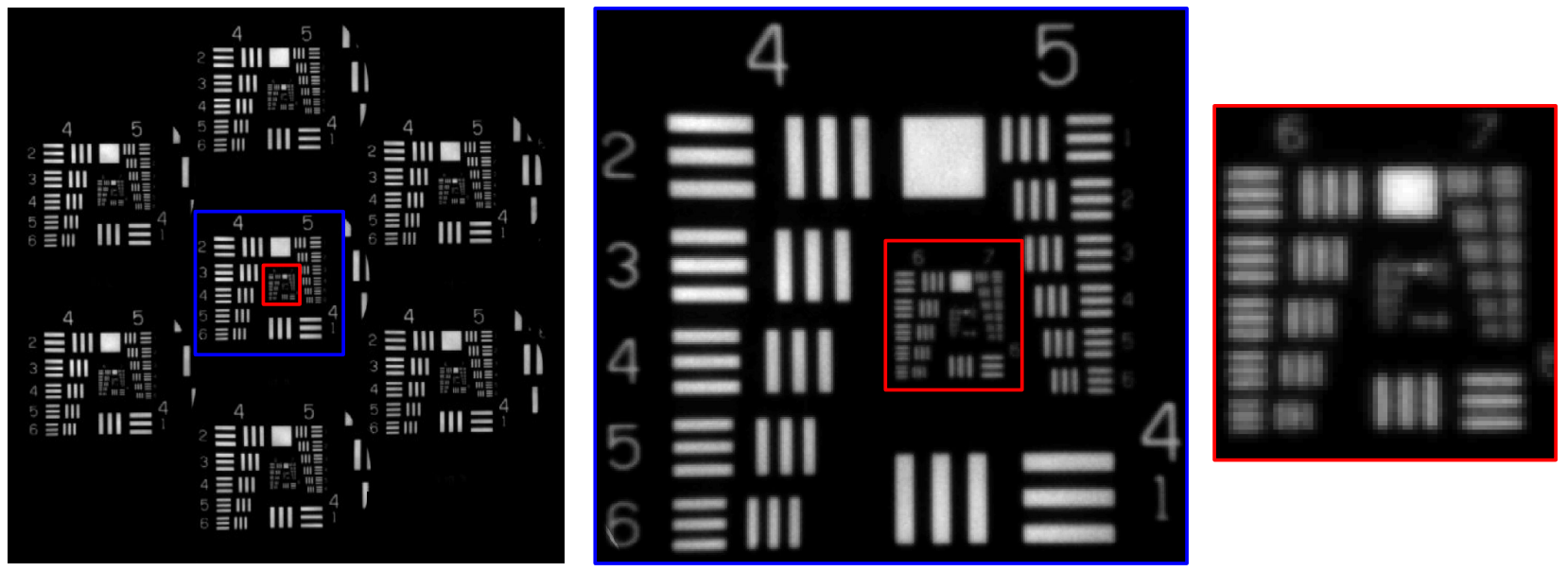

Figure 2. Left: Raw light field image of the USAF-1951 resolution target captured with our experimental FiMic setup. Center: Central elemental image. Right: Zoomed-in image of groups 6 and 7 of the USAF target.

We reconstructed the resolution target using both the MLEM algorithm and the OSL-TV algorithm to demonstrate the benefits of the regularization when performing deconvolution of FiMic data. For both schemes, we performed 100 iterations. Figure 3 (left) displays the result of the MLEM algorithm, where the improvement over the raw central EI in Figure 2 are evident. The deconvolved image resolves up to element 7.1 as shown in the close-up. However, the MLEM reconstruction suffers from artifacts, as emphasized in the close-ups. In Figure 3 (right) the OSL-TV manages to remove these artifacts producing smooth homogeneous regions (see the white squared region), while preserving the sharp details of the image (element 7.1 is still resolved). We encourage the reader to zoom into the figure for a better visual comparison.

\section{CONCLUSION}

In this work we proposed a deconvolution method for FiMic light field images, based on the well known Expectation-Maximization algorithm and augmented with total variation regularization to deal with noise. We demonstrated, using experimental images, the proposed method produces superior reconstruction results over the state of the art deconvolution by removing the noise artifacts while preserving the sharp details in the data.

\section{IMPLEMENTATION}

The implementation of the methods described in this paper are available as part of our 3D reconstruction framework for light field microscopy oLaF, available at: https://gitlab.lrz.de/IP/olaf. ${ }^{41}$ 

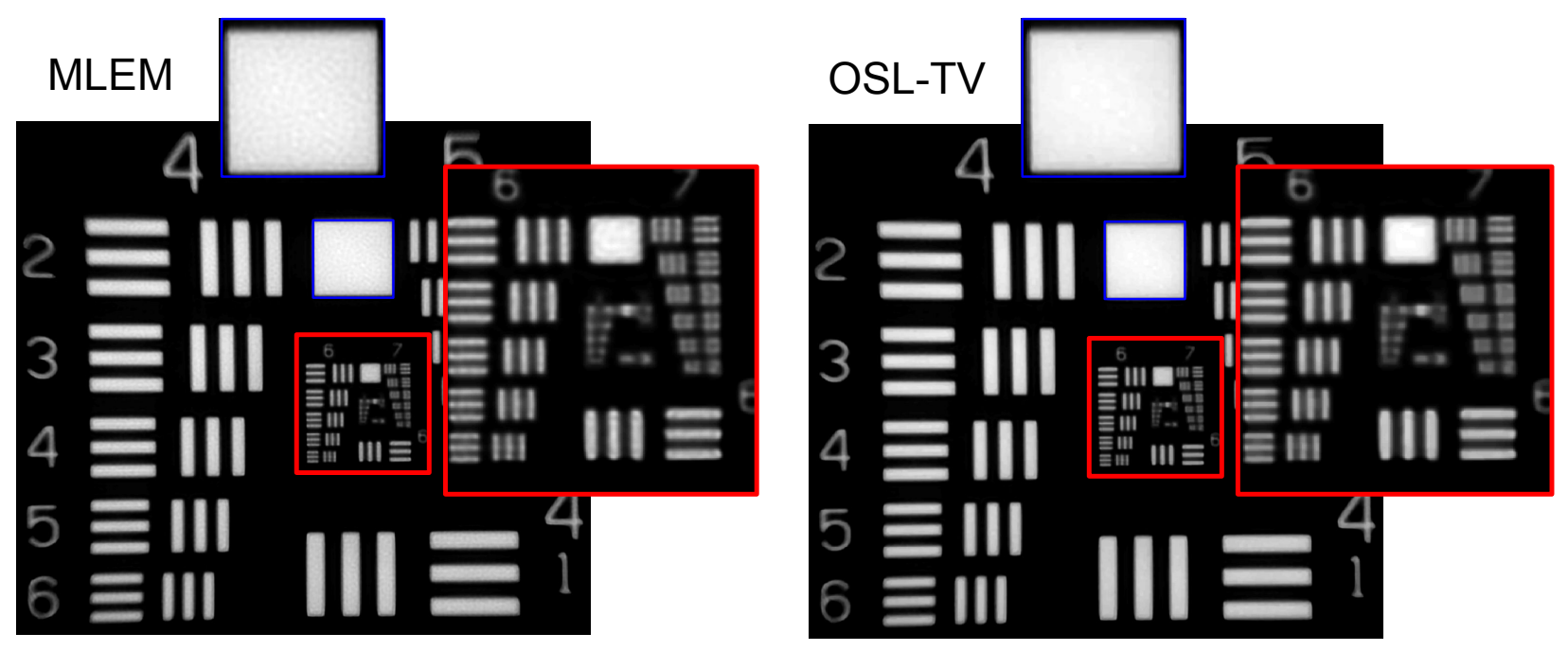

Figure 3. Left: The MLEM reconstruction of the USAF resolution target resolved up to element 7.1. While, the deconvolved image shows an overall improved appearance over the raw light field image in Figure 2, it exhibits artifacts as highlighted in the close-up regions. Right: The OSL-TV $(\lambda=0.001)$ produces a smooth reconstruction, while preserving the sharp discontinuities as expected.

\section{ACKNOWLEDGMENTS}

This work was funded by the German Research Foundation (DFG LA 3264/2-1), Ministerio de Ciencia, Innovación y Universidades, Spain (Grant RTI2018-099041-B-I00), and Generalitat Valenciana, Spain (Grant PROMETEO/2019/048).

\section{REFERENCES}

[1] Adelson, E. H. and Wang, J. Y. A., "Single Lens Stereo with a Plenoptic Camera," Tech. Rep. 2 (1992).

[2] Lippmann, G., "Épreuves Réversibles Donnant La Sensation Du Relief," Journal de Physique Théorique et Appliquée 7(1), 821-825 (1908).

[3] Ng, R., Levoy, M., Brédif, M., Duval, G., Horowitz, M., and Hanrahan, P., "Light Field Photography with a Hand-Held Plenoptic Camera - Stanford Tech Report CTSR 2005-02," tech. rep. (2005).

[4] Lumsdaine, A. and Georgiev, T., "The focused plenoptic camera," in [2009 IEEE International Conference on Computational Photography, ICCP 09], 1-8, IEEE (apr 2009).

[5] Georgiev, T. and Lumsdaine, A., "The multifocus plenoptic camera," Proceedings of SPIE 8299(1), 829908829911 (2012).

[6] Perwass, C. and Wietzke, L., "Single lens 3D-camera with extended depth-of-field," 829108 (2012).

[7] Bishop, T. E. and Favaro, P., "The light field camera: Extended depth of field, aliasing, and superresolution," IEEE Transactions on Pattern Analysis and Machine Intelligence 34(5), 972-986 (2012).

[8] Levoy, M., Ng, R., Adams, A., Footer, M., and Horowitz, M., "Light field microscopy," ACM Transactions on Graphics 25(3), 924-934 (2006).

[9] Llavador, A., Sola-Pikabea, J., Saavedra, G., Javidi, B., and Martínez-Corral, M., "Resolution improvements in integral microscopy with Fourier plane recording," Optics Express 24(18), 20792-20798 (2016).

[10] Llavador, A., Garcia-Sucerquia, J., Sanchez-Ortiga, E., Saavedra, G., and Martinez-Corral, M., "View images with unprecedented resolution in integral microscopy," OSA Continuum 1(1), 40-47 (2018).

[11] Martinez-Corral, M. and Javidi, B., "Fundamentals of 3D imaging and displays: a tutorial on integral imaging, light-field, and plenoptic systems," Advances in Optics and Photonics 10(3), 512-566 (2018). 
[12] Ng, R., "Fourier slice photography," in [ACM SIGGRAPH 2005 Papers on - SIGGRAPH '05], 735-744 (2005).

[13] Dansereau, D. G., Pizarro, O., and Williams, S. B., "Linear Volumetric Focus for Light Field Cameras," ACM Transactions on Graphics 34(2), 1-20 (2015).

[14] Bishop, T. E., Zanetti, S., and Favaro, P., "Light field superresolution," in [2009 IEEE International Conference on Computational Photography (ICCP)], 1-9 (2009).

[15] Tao, M. W., Hadap, S., Malik, J., and Ramamoorthi, R., "Depth from combining defocus and correspondence using light-field cameras," in [Proceedings of the IEEE International Conference on Computer Vision], 673-680 (2013).

[16] Tao, M. W., Su, J. C., Wang, T. C., Malik, J., and Ramamoorthi, R., "Depth Estimation and Specular Removal for Glossy Surfaces Using Point and Line Consistency with Light-Field Cameras," IEEE Transactions on Pattern Analysis and Machine Intelligence 38(6), 1155-1169 (2016).

[17] Broxton, M., Grosenick, L., Yang, S., Cohen, N., Andalman, A., Deisseroth, K., and Levoy, M., "Wave optics theory and 3-D deconvolution for the light field microscope," Optics Express 21(21), 25418-25439 (2013).

[18] Stefanoiu, A., Page, J., Symvoulidis, P., Westmeyer, G. G., and Lasser, T., "Artifact-free deconvolution in light field microscopy," Optics Express 27(22), 31644-31666 (2019).

[19] Lu, Z., Wu, J., Qiao, H., Zhou, Y., Yan, T., Zhou, Z., Zhang, X., Fan, J., and Dai, Q., "Phase-space deconvolution for light field microscopy," Optics Express 27(13), 18131 (2019).

[20] Prevedel, R., Yoon, Y. G., Hoffmann, M., Pak, N., Wetzstein, G., Kato, S., Schrödel, T., Raskar, R., Zimmer, M., Boyden, E. S., and Vaziri, A., "Simultaneous whole-animal 3D imaging of neuronal activity using light-field microscopy," Nature Methods 11, 727-730 (jul 2014).

[21] Wagner, N., Norlin, N., Gierten, J., de Medeiros, G., Balázs, B., Wittbrodt, J., Hufnagel, L., and Prevedel, R., "Instantaneous isotropic volumetric imaging of fast biological processes," Nature Methods 16(6), 497-500 (2019).

[22] Page, J., Saltarin, F., Belyaev, Y., Lyck, R., and Favaro, P., "Learning to Reconstruct Confocal Microscopy Stacks from Single Light Field Images," (2020).

[23] Li, H., Guo, C., Kim-Holzapfel, D., Li, W., Altshuller, Y., Schroeder, B., Liu, W., Meng, Y., French, J. B., Takamaru, K.-I., Frohman, M. A., and Jia, S., "Fast, volumetric live-cell imaging using high-resolution light-field microscopy," Biomedical Optics Express 10(1), 29-49 (2019).

[24] Levoy, M., Zhang, Z., and McDowall, I., "Recording and controlling the 4D light field in a microscope using microlens arrays," Journal of Microscopy 235(2), 144-162 (2009).

[25] Liang, C.-K. and Ramamoorthi, R., "A Light Transport Framework for Lenslet Light Field Cameras," $A C M$ Trans. Graph. 34(2) (2015).

[26] Cohen, N., Yang, S., Andalman, A., Broxton, M., Grosenick, L., Deisseroth, K., Horowitz, M., and Levoy, M., "Enhancing the performance of the light field microscope using wavefront coding," Optics Express 22, 24817- 24839 (oct 2014).

[27] Lu, C.-H., Muenzel, S., and Fleischer, J., "High-Resolution Light-Field Microscopy," in [Imaging and Applied Optics], CTh3B.2 (2013).

[28] Scrofani, G., Sola-Pikabea, J., Llavador, A., Sanchez-Ortiga, E., Barreiro, J. C., Saavedra, G., GarciaSucerquia, J., and Martinez-Corral, M., "FIMic: design for ultimate 3D-integral microscopy of in-vivo biological samples," Biomedical Optics Express 9(1), 335-346 (2018).

[29] Guo, C., Liu, W., Hua, X., Li, H., and Jia, S., "Fourier light-field microscopy," Optics Express 27(18), 25573-25594 (2019).

[30] Cong, L., Wang, Z., Chai, Y., Hang, W., Shang, C., Yang, W., Bai, L., Du, J., Wang, K., and Wen, Q., "Rapid whole brain imaging of neural activity in freely behaving larval zebrafish (Danio rerio)," eLife 6 (2017).

[31] Richardson, W. H., "Bayesian-Based Iterative Method of Image Restoration," Journal of the Optical Society of America 62(1), 55-59 (1972).

[32] Lucy, L. B., "An iterative technique for the rectification of observed distributions," The Astronomical Journal 79, 745 (1974). 
[33] Dempster, A. P., Laird, N. M., and Rubin, D. B., "Maximum Likelihood from Incomplete Data Via the EM Algorithm," Journal of the Royal Statistical Society: Series B (Methodological) 39(1), 1-22 (1977).

[34] Shepp, L. A. and Vardi, Y., "Maximum Likelihood Reconstruction for Emission Tomography," IEEE Transactions on Medical Imaging 1(2), 113-122 (1982).

[35] Green, P. J., "On Use of the Em Algorithm for Penalized Likelihood Estimation," Journal of the Royal Statistical Society: Series B (Methodological) 52(3), 443-452 (1990).

[36] Rudin, L. I., Osher, S., and Fatemi, E., "Nonlinear total variation based noise removal algorithms," Physica D: Nonlinear Phenomena 60(1-4), 259-268 (1992).

[37] Dey, N., Blanc-Feraud, L., Zimmer, C., Roux, P., Kam, Z., Olivo-Marin, J. C., and Zerubia, J., "RichardsonLucy algorithm with total variation regularization for 3D confocal microscope deconvolution," Microscopy Research and Technique 69(4), 260-266 (2006).

[38] Shroff, S. A. and Berkner, K., "Image formation analysis and high resolution image reconstruction for plenoptic imaging systems," Applied Optics 52(10), D22-D31 (2013).

[39] Getreuer, P., "Rudin-Osher-Fatemi Total Variation Denoising using Split Bregman," Image Processing On Line 2, 74-95 (2012).

[40] Chambolle, A., "An Algorithm for Total Variation Minimization and Applications," in [Journal of Mathematical Imaging and Vision], 20(1-2), 89-97 (2004).

[41] Stefanoiu, A., Page, J., and Lasser, T., "olaf: A flexible 3d reconstruction framework for light field microscopy," tech. rep. (2019). 